\title{
Test in applications with regulatory requirements on the example of WLTP
}

Dr. Jan Daniel Jacob

Werum Software \& Systems AG

This manuscript is not available according to publishing restriction. Thank you for your understanding. 